\title{
LIMITED WEGENER'S GRANULOMATOSIS AS A SOLITARY PULMONARY NODULE - A CASE REPORT
}

\author{
Mishra $\mathrm{M}^{1}$, Singla $\mathrm{R}^{2}$, Dewan $\mathrm{RK}^{3}$ \\ ${ }^{1}$ Subharti Medical College, Meerut - 250 005, U.P., India \\ ${ }^{2,3}$ LRS Institute of TB \& Respiratory Diseases, Sri Aurobindo Marg, New Delhi -110 030, India
}

\begin{abstract}
Limited Wegener`s Granulomatosis (WG) has been infrequently reported from India so far. We report a case of Limited WG in a 60 year old male, who presented to us with productive cough and hemoptysis, and a right upper lobe mass lesion on chest roentgenogram. He was previously treated outside as Pulmonary Koch's, without any improvement. We diagnosed the patient on histo-pathological examination of right upper lobectomy specimen. He was administered five pulses of intravenous cyclophosphamide therapy plus oral corticosteroids, with significant symptomatic improvement. He was given oral methotrexate and tapering doses of corticosteroids in follow up.
\end{abstract}

Key words: Limited Wegener's Granulomatosis, Solitary Pulmonary Nodule

\section{INTRODUCTION}

Wegener's granulomatosis is a systemic necrotizing granulomatous vasculitis of unknown etiology, which usually consists of a triad of necrotizing angiitis of the upper and lower respiratory tract and focal glomerulonephritis of the kidney (classicl severe form) $)^{1,2}$, but may virtually involve any organ system. The concept of limited form of Wegener's was introduced in 1966 by Carrington and Liebow ${ }^{3}$ to identify otherwise classic vasculitis lacking renal involvement. Limited Wegener's is said to carry a better prognosis than classic disease ${ }^{2,4}$, but it may be extremely challenging to recognize and diagnose. Anti-neutrophil cytoplasmic antibodies (ANCAs) have a high degree of association with WG. Their positive predictive value ranges between $19-66 \%{ }^{2,5}$ c-ANCA is more specific than p-ANCA and is positive in more than $90 \%$ of patients with active WG. ${ }^{6}$ In Limited Wegener's, the sensitivity of c-ANCA is $65-70 \%{ }^{6}$ Those patients of WG who

\section{Correspondence:}

Dr. Rupak Singla

Head, Department of TB \& Respiratory Diseases LRS

Institute of TB \& Respiratory Diseases

Sri Aurobindo Marg, New Delhi-110030, India.

Phone: (+91)9891168908 are $\mathrm{p}$-ANCA positive $(<5 \%)^{7}$ have fewer organ involvement than those with c-ANCA.

\section{CASE REPORT}

We report the case of a 60 year old normotensive, non-diabetic male, ex-smoker, who was referred to us as a suspected case of right upper lobe lung abscess/aspergilloma for surgical opinion. This gentleman presented to us with complaints of shortness of breath on exertion for last three years, cough with expectoration of purulent sputum for last ten months, and streaking of sputum, off and on, for last ten months.

Previously, about 30 years back, the patient took Anti-Tubercular Therapy (ATT) for left pleural effusion for one year. For chronic cough, streaking of sputum and suggestive radiological picture, he again received ATT from outside for ten months, nearly a year before admission to our institute in November 2009.

CT Thorax (Figure 1) revealed a well defined cystic lesion $3.74 \mathrm{~cm} \times 4.69 \mathrm{~cm}$ in the apical segment of right upper lobe with mild surrounding consolidation, with an eccentric air shadow seen within it. No mediastinal lymph nodes were seen. IgG-hydatid cyst (2.37 U/L), total IgE (189 IU/L) and serum 
precipitins against Aspergillus were negative. A CT guided FNA of the mass lesion revealed necrotic cell debris and degenerated inflammatory cells; no epithelioid cells/AFB were visualized.

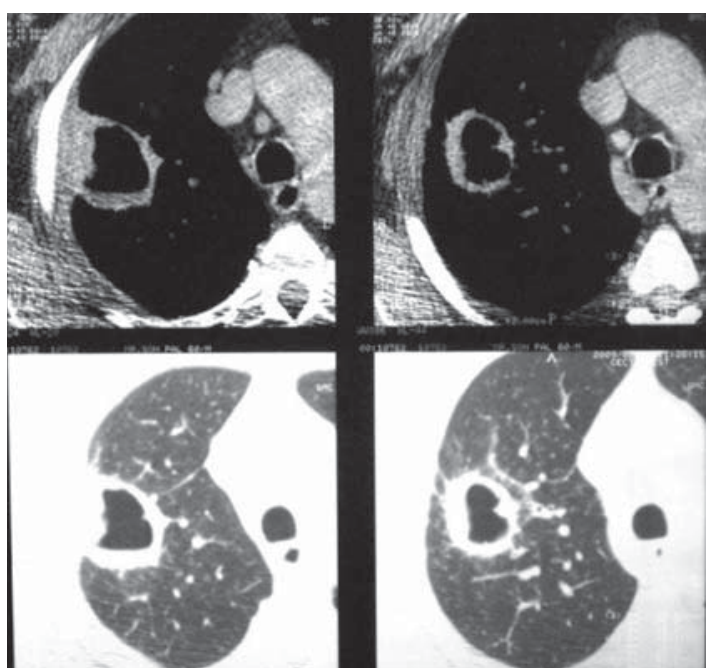

Figure 1: CT-Thorax showing well defined cystic lesion $3.74 \mathrm{~cm} \times 4.69 \mathrm{~cm}$ in the apical segment of right upper lobe

With the above previous investigations that were performed outside, the patient was referred to our institute for further evaluation. The general physical examination was unremarkable, and systemic examination did not reveal any significant abnormality, except for occasional wheeze heard upon auscultation of the chest. There was no history of dysuria, oliguria, hematuria; allergy, nasal discharge, recurrent ENT infection, hearing difficulty, skin or eye lesions; joint pains, hematemesis, malena, abdominal pain; orthopnea, palpitations, or swelling of feet / face. All routine baseline investigations viz. hemogram, liver function tests, renal function tests, electrolytes, $\mathrm{HIV} / \mathrm{HBsAg} / \mathrm{HCV}$ and urine routine/microscopic examination were within normal limits. The chest $x$-ray (Figure 2) showed a mass lesion with an air-fluid level in the right upper lobe. A right upper lobectomy was performed which revealed a welldefined cystic lesion in right upper lobe. Histopathological examination of the resected specimen was suggestive of necrotizing granulomatous lesion associated with vasculitis, possibly Wegener's Granulomatosis. c-ANCA was weakly positive. The patient was diagnosed as a case of limited Wegener`s Granulomatosis.

Between December 2009 and April 2010, the patient was administered five cycles of monthly pulse cyclophosphamide therapy with injection MESNA [2-Mercapto Ethane Sulfonate sodium (NA)] and oral corticosteroids. Thereafter he was given oral methotrexate with tapering dose of corticosteroids. Over the course of his treatment, he showed significant symptomatic relief. Subsequently he was lost to follow-up after six months.

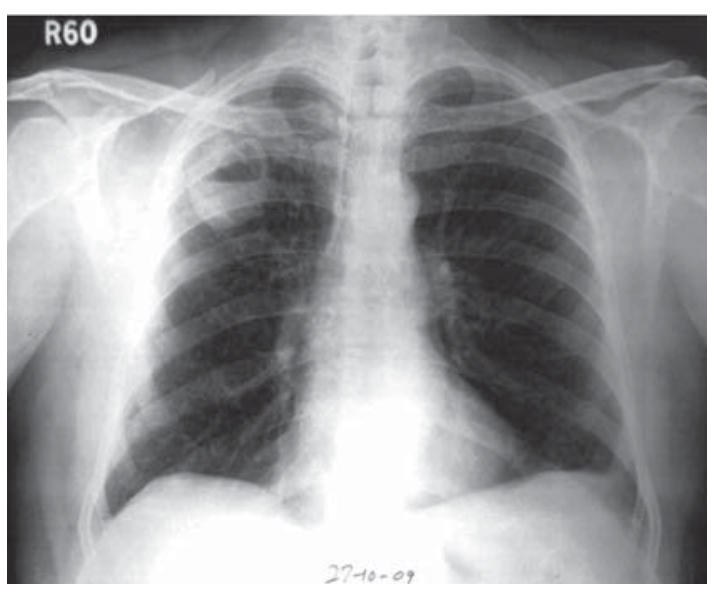

Figure 2: Chest $X$-ray PA view showing mass lesion with an air-fluid level in the right upper lobe

\section{DISCUSSION}

WG is the most frequent ANCA-associated vasculitis. Clinically, it is characterized by the triad of upper airway disease (otitis, ulcerations, sinusitis, bony deformities, and tracheal or bronchial stenosis), lower respiratory tract involvement (chest pain, cough, dyspnoea, and hemoptysis), and glomerulonephritis, which occurs in 50-90\% patients. ${ }^{8-11}$ Upper airway disease is the most common presenting feature of WG, sinusitis being the most frequent initial presentation in about half to two thirds of patients. ${ }^{12,13}$. Pulmonary involvement occurs in $45 \%$ of patients at presentation and in more than $90 \%$ of patients during the course of the disease. In $9 \%$ of the cases, lung is the only organ affected. ${ }^{14}$ Lung involvement may range from subclinical changes (found as incidental findings in high-resolution CT scans), to the finding of diffuse alveolar hemorrhage in the most devastating cases. ${ }^{15}$ In the mid-1960s, limited forms of WG were recognized. Limited disease, in contrast to the severe form, includes manifestations of WG that pose no immediate threat to either the patient's life or the function of a vital organ. ${ }^{4}$ Advanced age, severe renal involvement, alveolar hemorrhage and C-ANCA positivity correlate with a worse outcome. ${ }^{16-18}$ 
Unlike other granulomatous disorders, eg. sarcoidosis and post-primary tuberculosis, WG has a tendency to involve the lower portions of the lungs. ${ }^{3}$ Radiological presentation may be in the form of interstitial, mixed, or alveolar

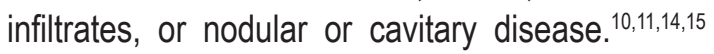
The most common radiographic findings include pulmonary infiltrate $(67 \%)$ and nodules $(58 \%) .^{12}$ Nodules are predominantly subpleural and are cavitated in nearly $50 \%$ of cases. ${ }^{19}$ In limited forms, the pulmonary lesions are usually multiple and bilateral, often simulate primary or metastatic neoplasms of the lung, but may suggest infectious granulomatous disease. Our case presented as a solitary pulmonary nodule.

Traditional initial therapy of WG consists of daily oral cyclophosphamide-corticosteroid combination therapy. This treatment has been quite effective in inducing remission in $>90 \%$ of patients. However, in patients who have limited disease, monthly intravenous cyclophosphamide pulse therapy can be effective and minimize the side effects. ${ }^{20-22}$ Pulse therapy is given in conjunction with prednisone, usually starting at $1 \mathrm{mg} / \mathrm{kg} / \mathrm{day}$ for at least 1 month, then tapering by 5 to $10 \mathrm{mg} /$ week until prednisone is stopped or the patient is placed on maintenance therapy of 5-15 mg every other day. Other therapies for WG include methotrexate and prednisone, which is an alternative for patients with active but not immediately life threatening disease and normal or near-normal renal function. ${ }^{23,24}$ Prednisone alone is not a recommended therapy for WG. ${ }^{22}$

Our patient illustrates an unusual presentation of histo-pathologically proven limited WG, in which the pulmonary lesion was located in the apical segment of right upper lobe as a solitary pulmonary nodule. Surgery (right upper lobectomy) was performed to establish a tissue diagnosis. No evidence of systemic vasculitis could be found. The differential diagnoses that we considered in our patient and their possible respective reasons for exclusion were:

1. Pulmonary tuberculosis - no improvement with ATT.

2. Lung abscess - no history of foul-smelling copious expectoration, any dental infection/ procedure, and no improvement with systemic antibiotics.
3. Fungal infection (aspergilloma) - total lgE and serum precipitins against aspergillus species were negative.

4. Neoplasm of the lung - chronic illness, without a history of weight loss and no evidence of metastases.

Cases of WG presenting with a solitary pulmonary nodule have been described, but uncommonly. ${ }^{25}$ This case report thus represents a rather uncommon presentation of limited Wegener's granulomatosis as a solitary pulmonary nodule in the apical segment of right upper lobe.

\section{CONCLUSION}

Limited WG is an uncommon entity that may pose significant diagnostic challenges as it closely mimics other more common disorders like pulmonary tuberculosis, lung cancer, lung abscess, etc. An improved knowledge of clinical and imaging features of WG could help the clinician reach an earlier diagnosis. Also, once correctly identified, the response to treatment as well as prognosis of limited WG is better as compared to severe WG. We therefore recommend having a high index of suspicion for this form of the disease, especially when the patient is inadequately responding to other forms of treatment. Future areas of research may include the investigation of other therapeutic regimens/alternative forms of therapy that could match the success of cyclophosphamide and at the same time is free from concerns about toxicity arising from prolonged usage. The difference between limited and severe forms of WG with regard to sex, age, likelihood of recurrent disease, risk of damage in certain organ systems, and, possibly, etiologic factors also needs further clinical investigations in order to explore the pathogenesis, therapeutic, and prognostic differences between these two subsets.

\section{REFERENCES}

1. Stone JH. Limited versus severe Wegener's granulomatosis: baseline data on patients in the Wegener's granulomatosis etanercept trial. Arthritis Rheum 2003:48:2299-309.

2. Mlika M, Ayadi-Kaddour A, Marghli A, Kilani T, El Mezni F. Pulmonary localization revealing Wegener's granulomatosis. Scientific World Journal 2010;10:806-10. 
3. Carrington $\mathrm{CB}$, Liebow A. Limited forms of angiitis and granulomatosis of Wegener's type. Am J Med 1966;41:497-527.

4. Reinhold-Keller E, Beuge N, Latza U, de Groot K, Rudert H, Nölle B et al. An interdisciplinary approach to the care of patients with Wegener's granulomatosis: long-term outcome in 155 patients. Arthritis Rheum 2000;43:1021-32.

5. Shafiei K, Luther E, Archie M, Gulick J, Fowler MR. Wegener granulomatosis: case report and brief literature review. J Am Board Fam Pract 2003;16:555-9.

6. Nolle B, Specs U, Ludemann J, Rohrbach MS, DeRemee RA, Gross WL. Anticytoplasmic autoantibodies: their immunodiagnostic value in Wegener's Granulomatosis. Ann Intern Med 1989;111:28-40.

7. Schonermarck U, Lamprecht $P, \quad$ Csernok E, Gross WL. Prevalence and spectrum of rheumatic diseases associated with proteinase 3-antineutrophil cytoplasmic antibodies (ANCA) and myeloperoxidase-ANCA. Rheumatology 2001;40:178-84.

8. Frankel SK, Cosgrove GP, Fischer A et al. Update in the diagnosis and management of pulmonary vasculitis. Chest 2006;29:452-65.

9. Frankel SK, Sullivan EJ, Brown KK. Vasculitis: Wegener granulomatosis, Churg-Strauss syndrome, microscopic polyangiitis, polyarteritis nodosa, and Takayasu arteritis. Crit Care Clin 2002;18:855-79.

10. Cordier J-F, Valeyre D, Guillevin L et al. Pulmonary Wegener's granulomatosis: a clinical and imaging study of 77 cases. Chest 1990;97:906-12.

11. Anderson G, Coles ET, Crane M et al. Wegener's granulomatosis: a series of 265 British cases seen between 1975 and 1985: a report by a subcommittee of the British Thoracic Society Research Committee. Q J Med 1992;83:427-38.

12. Hoffman GS, Kerr GS, Leavitt RY et al. Wegener granulomatosis: an analysis of 158 patients. Ann Intern Med 1992;116:488-98.

13. Fauci AS, Haynes BF, Katz P, Wolff SM. Wegener's granulomatosis: prospective clinical and therapeutic experience with 85 patients for 21 years. Ann Intern Med 1983;98:76-85.

14. Thickett DR, Richter AG, Nathani $\mathrm{N}$ et al. Pulmonary manifestations of anti-neutrophil cytoplasmic antibody (ANCA)-positive vasculitis. Rheumatology 2006;45:261-8.
15. Schnabel A, Reuter M, Csernok E et al. Subclinical alveolar bleeding in pulmonary vasculitides: correlation with indices of disease activity. Eur Respir J 1999;14:118-24.

16. Bligny D, Mahr A, Toumelin PL et al. Predicting mortality in systemic Wegener's granulomatosis: a survival analysis based on 93 patients. Arthritis Rheum 2004;51:83-91.

17. Neuman I, Kain R, Regele H et al. Histological and clinical predictors of early and late renal outcome in ANCA-associated vasculitis. Nephrol Dial Transplant 2005;20:96-104.

18. Gayraud M, Guillevin L, le Toumelin P et al. Longterm follow up of polyarteritis nodosa, microscopic polyangiitis, and Churg-Strauss syndrome: analysis of four prospective trials including 278 patients. Arthritis Rheum 2001;44:666-75.

19. Courthaliac C, Aumaítre O, Andre' M et al. Apport de la tomodensitome' trie dans le suivi e' volutif des le' sions pleuropulmonaires de la granulomatose de Wegener. Rev Med Interne 1999;20:571-8.

20. Guillevin L, Cordier J-F, Lhote F et al. A prospective multicenter randomized trial comparing steroids and pulse cyclophosphamide versus steroids and oral cyclophosphamide in the treatment of generalized Wegener's granulomatosis. Arthritis Rheum 1997;40:2187-98.

21. Haubitz M, Schellong S, Gobel U et al. Intravenous pulse administration of cyclophosphamide versus daily oral treatment in patients with antineutrophil cytoplasmic antibody-associated vasculitis and renal involvement: a prospective randomized study. Arthritis Rheum 1998;41:1835-44.

22. de Groot K, Adu D, Savage CO. The value of pulse cyclophosphamide in ANCA-associated vasculitis: meta-analysis and critical review. Nephrol Dial Transplant 2001;16:2018-27.

23. Langford CA, Sneller MC, Hoffman GS. Methotrexate use in systemic vasculitis. Rheum Dis Clin North Am 1997;23:841-53.

24. Langford CA, Talar-Williams C, Sneller MC. Use of methotrexate and glucocorticoids in the treatment of Wegener's granulomatosis. Long-term renal outcome in patients with glomerolonephritis. Arthritis Rheum 2000;43:1836-40.

25. Papiris SA, Manoussakis MN, Drosos AA, Kontogiannis D, Constantopoulos SH, Moutsopoulos HM. Imaging of thoracic Wegener's granulomatosis: the tomographic appearance. Am J Med 1992;93:529-36. 